\title{
Aplicación de la cadena total de abastecimiento en las empresas peruanas
}

\author{
Luis Ernesto De Olazával Tejada \\ Universidad de Lima \\ Ingeniería Industrial n. 28, 2010, ISSN 1025-9929, pp.11-21 \\ Recibido: 24/09/2009 / Aprobado: 6/05/2010
}

\begin{abstract}
RESUMEN: La creciente globalización de la economía, la aparición en el mercado de nuevas tecnologías y la velocidad con que estas se actualizan, la preocupación del Gobierno por mejorar las relaciones internacionales mediante las firmas de los tratados de libre comercio (TLC) son causas suficientes para que nuestras empresas den un giro de 180 grados y realicen un análisis de su situación actual, con la finalidad de buscar la mejora continua que les permita ser cada vez más competitivas. Esta ansiada competitividad la lograrán con la formación de alianzas estratégicas con sus proveedores y sus clientes, características principales de la cadena total de abastecimiento (SCM por sus siglas en inglés).
\end{abstract}

Palabras clave: cadena total de abastecimiento / globalización / competitividad / logística integral

\section{Supply chain management in Peruvian enterprices}

ABSTRACT: Economy globalization, appearance of new technology in our market and the way they are being upgrade, the concern of our government to improve our international trading relationships with other countries, in which our treatise of free trade are the fact to our enterprises to make a switch and analyze their situation to be more competitive. However, they need agreements with clients and providers; main features for supply chain management.

Keywords: supply chain management / globalization / competitiveness / integral logistic 


\section{INTRODUCCIÓN}

El presente artículo es el resultado de una investigación llevada a cabo por la Escuela de Negocios de la Universidad de Lima, sobre la aplicación de la cadena total de abastecimientos en las empresas peruanas. El año que se realizó la investigación (2004-2005), nuestro país vivía una realidad diferente a la actual: la mayoría de las empresas trabajaba con una capacidad de planta menor al 50\%, que en algunos casos las llevó al cierre de sus plantas. Bastaba recorrer la avenida Argentina para ver ese aspecto desolador de nuestras industrias. Por otro lado, la importación de productos de dudosa calidad, en su gran mayoría procedente de China, el ingreso al país de productos subvaluados (dumping) con el consiguiente perjuicio para la industria nacional, un mercado interno reducido, entre otros problemas, daban un tinte especial al panorama nacional de ese entonces.

La tecnología de la información viajaba a una velocidad asombrosa, aparecían en el mercado variedad de programas informáticos que buscaban la mecanización y la integración de la totalidad de funciones de una empresa. El tiempo de vida promedio de los productos electrónicos (de procedencia importada) se reducía considerablemente. Por otro lado, el avance de la tecnología en el mundo ha generado la aparición de nuevos sistemas tendientes a la optimización de los procesos, desde la creación de un plan de necesidades, cualquiera sea el horizonte, hasta la satisfacción del cliente final.

En ese entonces se iniciaron las conversaciones para la firma de nuevos tratados internacionales, como el TLC con Estados Unidos; se produjo el ingreso a mercados de la región como el Mercosur, se mejoraron las relaciones comerciales con Chile, se abrieron los mercados del Asia, etcétera. Todas estas acciones, destinadas a buscar nuevos y mejores mercados para nuestros productos, daban a las empresas exportadoras peruanas, gracias al aumento de la demanda, la posibilidad de incrementar sus exportaciones. Pero para ello necesitábamos realizar a fondo un estudio de las exigencias de los futuros clientes y compararlas con nuestra realidad.

Si bien se incrementaban las posibilidades de apertura de nuevos mercados, también era cierto que para lograr una presencia viva en estos teníamos que competir contra otros países de la región, que estaban en la misma o mejor situación que la nuestra. 
Esta investigación busca conocer en qué medida las empresas nacionales estaban preparadas para dar el gran salto hacia la integración, la modernidad y, por ende, hacia la competitividad.

Hoy la situación de nuestro país es totalmente diferente, hemos tenido un crecimiento económico promedio anual del 8\%, que debido a la crisis financiera internacional se redujo a cifras que bordeaban el $1 \%$. Sin embargo, para el año 2010 se tiene pensado crecer en promedio en 4\% anual. Debemos recordar también que el 2008 se realizaron en el Perú dos eventos de suma importancia para nuestro desarrollo: la reunión de países de América Latina, el Caribe y la Unión Europea (Alcue) y el Foro de Cooperación Económica Asia Pacífico (APEC, por sus siglas en inglés).

Por otro lado, la firma de los TLC con Estados Unidos y China, las negociaciones que se llevan a cabo con la Comunidad Económica Europea, y las que se realizan para un próximo TLC con Corea del Sur y Japón, son indicadores de que se avecinan nuevos y mejores vientos para el país, y por lo tanto las empresas peruanas deben estar preparadas para dar ese gran salto hacia el futuro.

Por ello, nuestra investigación recobra actualidad y podría ser aplicada, sin lugar a dudas, a todas las empresas, en especial a las pymes. El éxito radica en esa incesante búsqueda de la mejora continua. Cabe señalar que la presente investigación ha sido resumida en cinco capítulos.

\section{ANÁLISIS DE LA INFORMACIÓN OBTENIDA A TRAVÉS DEL LEVANTAMIENTO DE DATOS}

Como parte de la investigación se realizaron encuestas con la finalidad de analizar de qué manera algunas empresas peruanas están iniciando el proceso de adaptación de sus organizaciones al uso de la cadena de abastecimientos (SCM, por su sigla en inglés), y, de haberlo hecho, examinar los resultados; asimismo, buscamos identificar los factores limitantes en el proceso de adecuación del SCM en estas empresas, y cómo se solucionaron.

Para el presente proyecto de investigación se consideró como población la totalidad de empresas existentes en las provincias de Lima y Callao, clasificadas en grandes, medianas y pequeñas. No se tomaron en cuenta las microempresas, pues a pesar de que representan el $80 \%$ 
del total de empresas existentes, no califican para la presente investigación, por sus problemas económicos, la reducida o escasa visión de empresa y su falta de tecnología, entre otros factores. De las 600 empresas contactadas solo respondieron a nuestra solicitud 48 empresas, con las siguientes características: grandes 10,40\%; medianas $35,40 \%$, y pequeñas $54,20 \%$.

La encuesta constó de cinco partes: organización, procesos, proveedores, clientes y cadena total de abastecimiento.

Se desarrollaron un total de 46 encuestas, cuyos resultados se indican a continuación.

\subsection{Organización}

Sobre la dependencia directa de la función logística, en el caso de las empresas de manufactura, hoteleras o comercializadoras, un 43,5\% depende funcionalmente de la Gerencia de Operaciones, que a su vez, según el tamaño de la empresa, está subordinada directamente a la Gerencia General. Un 23,9\% informó que está condicionada funcionalmente a la Gerencia de Administración.

Las dependencias que componen la función logística en el caso de las mismas empresas son: compras, almacenes y control de inventarios. La dependencia de catalogación la consideran como una función principal de la logística a cargo de control de inventarios.

Acerca de las actividades de tercerización de la función logística, un alto porcentaje de empresas ha optado por tercerizar las funciones de transportes y aduanas. Sin embargo, existe también un pequeño porcentaje que han optado por lo propio con otras funciones, como las de compras y almacenes.

Sobre la utilización de los servicios de un operador logístico, el $43,5 \%$ de las empresas informa que contrata estos servicios, principalmente para las funciones de trámite de importación (31,4\%), fletes $(31,4 \%)$ y en menor escala, para almacenes $(25,7 \%)$ y distribución física $(11,4 \%)$.

Es importante destacar que el $28,3 \%$ de las empresas que contratan los servicios de un operador logístico los califican como bueno y el $13,0 \%$ como muy bueno. Solo un $2,2 \%$ lo califica como regular. 


\subsection{Procesos}

Acerca del tiempo transcurrido entre los procedimientos de revisión o actualización de procesos en la empresa, el $46 \%$ de las empresas encuestadas afirma que la mejora o actualización es un proceso continuo. Sin embargo, aún queda un $31 \%$ que actualiza los procesos anualmente, o lo que es más grave aún: casi nunca.

En lo referente a si en la actualidad las empresas utilizan algún software para el proceso de compra, el 82,6\% de las empresas encuestadas contestó afirmativamente.

Del 17,4\% de empresas encuestadas que contestó no utilizar un software, el $49 \%$ no lo usa por no tener los recursos necesarios y el $36 \%$ por no contar con el personal adecuado.

Acerca de la antigüedad del software utilizado, del 82,6\% de empresas que sí utiliza un software para el proceso de compra el $39 \%$ tiene una antigüedad mayor de tres años y solo un $17,4 \%$ aún no cuenta con un software para manejar el proceso de compra.

De la totalidad de las empresas que sí usa software en el proceso de compra, un $41 \%$ no considera el control y la evaluación de proveedores, requisito importante contemplado en el SCM. Es pertinente resaltar también que del total de empresas que usan un software, el $67 \%$ tiene las características de un programa integrador, del tipo de planteamiento de recursos empresariales (ERP).

\subsection{Proveedores}

En esta tercera sección de preguntas se recopiló información acerca del trato con los proveedores que utiliza la empresa actualmente.

En el caso de las empresas privadas es interesante observar que las modalidades de cotización que más utilizan para establecer contacto con el proveedor es la vía telefónica y el correo electrónico, ambos con un $93 \%$.

Con relación a los factores que se analizan para tomar la decisión de elegir al ganador de la buena pro, podemos decir que del total de empresas encuestadas, han elegido como el más importante: 


\begin{tabular}{lc}
\hline Factor & Empresas (\%) \\
\hline Calidad & 80 \\
Precio & 20 \\
Cantidad & 36 \\
Tiempo de entrega & 24 \\
Lugar de entrega & 4 \\
Forma de pago & 11 \\
\hline
\end{tabular}

Esto nos da una idea clara de que cada vez son más las empresas que exigen mayor calidad a los proveedores como el factor más importante, determinante en el proceso de mejora continua.

Con relación a si en la mayoría de contratos por compra de bienes se solicita a los proveedores entrega parcial o total, el $62,2 \%$ de empresas informó que las entregas son del $100 \%$ de lo solicitado y según la fecha programada. Por otro lado, el 53\% de empresas encuestadas viene aplicando, en la mayoría de los contratos con proveedores, las entregas parciales según sus necesidades.

Teniendo en consideración que una de las tareas más importantes del Departamento de Compras es la de buscar al proveedor adecuado, y sabiendo además que la forma de optimizar el uso de los recursos de la empresa al desarrollar los procedimientos de compra es utilizar un registro de proveedores previamente seleccionados, el 93\% de las empresas encuestadas tiene el concepto claro de la importancia de mantener un registro de proveedores con esas características. De este total, un alto porcentaje (54\%) opina que su actualización es un proceso continuo.

Con relación a la manera como las empresas obtienen la relación de proveedores para su proceso de compra, en el caso de las empresas que no cuentan con un registro de proveedores la mayoría de ellas $(66,7 \%)$ obtiene información sobre proveedores a través de referencias, o son proveedores conocidos en el mercado. El 47,8\% de empresas responde que la actualización de proveedores es un proceso dinámico. Por otro lado, del total de empresas encuestadas el $43 \%$ informa que el contacto con sus proveedores es constante.

Lo que sí podemos calificar como beneficioso es el hecho de que un alto porcentaje de empresas se comunica con sus proveedores mediante el teléfono o a través del correo electrónico, lo que constituye un gran paso hacia el proceso del reabastecimiento automático y continuo. 
Acerca de la participación de los proveedores en la confección de los planes y programas de las empresas clientes, el resultado de la encuesta arrojó que es cada vez mayor, y con tendencia a incrementarse, en la medida en que ambos - proveedor y cliente - sientan la necesidad de trabajar en forma conjunta.

Del total de empresas encuestadas, el 43,2\% indica que mantiene entre 1 y 3 proveedores por familia de códigos, y un $37 \%$ indica que mantiene entre 4 y 6 proveedores.

Con relación a si las empresas exigen a sus proveedores certificación de la calidad a través de empresas especializadas, como requisito para ser parte de su registro de proveedores, el $69,6 \%$ de empresas encuestadas contestaron negativamente.

En el caso de las empresas que indicaron que no exigen certificación de la calidad a sus proveedores (30,4\%), un 70\% informó que comprueba la calidad a través de la información de otras empresas o visitas a las instalaciones de los proveedores. Un 30\% indicó que lo hace únicamente a través de la confianza.

\subsection{Clientes}

Con referencia a la participación de los clientes internos en la elaboración del plan empresarial, un $78,3 \%$ de las empresas encuestadas contesta afirmativamente, lo que demuestra que cada vez estamos tomando mayor conciencia de la importancia de participar activamente, pensando que somos un conjunto integrado y no entes aislados.

Este cambio de mentalidad se ve reflejado también en el hecho de mantener informados a los responsables logísticos cada vez que se presentan cambios en el comportamiento de la demanda.

Con relación a si los cambios en el comportamiento de la demanda son comunicados por el cliente, un $71,7 \%$ de empresas indica que sí.

Acerca de si el factor de servicio al cliente interno se mide por la calidad de los materiales o el comportamiento en la llegada de estos, la mayoría de empresas (62\%) afirma que el factor de servicios al cliente interno se mide por la oportunidad en la llegada de los materiales solicitados, bajo las características de calidad, cantidad y precios competitivos. Sobre este aspecto, solo un $38 \%$ afirma que el factor de servicio se mide por el grado de intercambio de la información en tiempo real. 


\subsection{Cadena total de abastecimiento}

Las preguntas contenidas en el último bloque de la encuesta están orientadas a comprobar si en los niveles gerenciales de la empresa se conocía el concepto de cadena total de abastecimiento y si era utilizado como herramienta para la optimización del logro de los objetivos de la organización.

Acerca de si las empresas tienen conocimiento sobre la existencia de la cadena total de abastecimiento, el $75 \%$ de las empresas encuestadas declara que sí tienen conocimiento de la existencia de la cadena total de abastecimiento y su utilización como herramienta para la optimización del logro de los objetivos de toda organización.

Del total de empresas que conoce la existencia de la cadena total de abastecimiento (75\%), el $79 \%$ indica que aplican los conocimientos del SCM en la optimización de recursos y un $73 \%$ en el intercambio de información con los proveedores y clientes.

En el caso de las empresas en que no existe interés respecto a la utilización de la cadena total de abastecimiento (25\%), el $72 \%$ indica que se debe a desconocimiento, mientras que solo un $8 \%$ señala la falta de capacitación del personal.

\section{CONCLUSIONES}

a) Estos resultados nos demuestran que el camino por recorrer es largo y está en nuestras manos generar los cambios necesarios que nos permitan hacer que nuestras empresas sean competitivas, $\mathrm{y}$ que logren una presencia viva tanto en el país como en los distintos mercados internacionales.

b) Hoy la competencia no es entre empresas sino entre cadenas. Cada eslabón que conforma una cadena representa una empresa, sean proveedoras, clientes, contratistas, financieras, aseguradoras, transportistas, etcétera. Estas empresas deben tener una característica común: la competitividad.

c) Es importante que todos estemos convencidos y comprometidos para impulsar a nuestras empresas hacia el cumplimiento de los objetivos y el logro de la tan ansiada competitividad, mediante el aporte de: 
- Voluntad de cambio.

- Iniciativa y creatividad.

- Compromiso.

- Compartir información.

- Asumir responsabilidades.

- Pensar que el cliente es el disparador de la cadena total de abastecimiento.

- Poder de negociación en la relación cliente-proveedor.

- Contratos a largo plazo con entregas parciales.

- Lograr confianza en la relación cliente-proveedor.

- Establecer una integración total tanto en dentro de la empresa como de esta con las demás empresas.

\section{RECOMENDACIONES}

a) El fenómeno de la globalización económica internacional y la política exterior del Gobierno peruano de impulsar los TLC, algunos ya firmados y otros en proceso de negociación, obligan a las empresas peruanas a dar un giro de 180 grados e iniciar un análisis profundo de sus instalaciones a fin de repotenciar sus fuerzas y solucionar sus debilidades, que les permitan hacer frente a la cada vez más creciente competencia.

b) El éxito de la cadena total de abastecimiento radica en el intercambio de información entre la totalidad de sus integrantes (relación cliente-proveedor) asumiendo cada uno la responsabilidad y el compromiso que le compete.

c) La logística es la responsable de la planificación y abastecimiento de las necesidades de la empresa y se fija como objetivo brindar un factor de servicio al cliente interno tendiente al 100\%. Para lograr ese cometido se interrelaciona con los clientes y los proveedores, formando parte de la cadena total de abastecimiento.

d) En la elaboración de un plan de compras es necesaria la interacción con proveedores de categoría potencial, que permita compartir información, y que los lleve hacia una negociación con beneficios para ambos (cliente-proveedor), con acuerdos de largo plazo y entregas parciales, entre otros.

e) Es importante que las empresas adquieran un software de características ERP que les permita la integración de todas las áreas internas y una interacción tanto con los proveedores de categoría 
potencial, como con los principales clientes de categoría A y B (según clasificación de Pareto sobre la base de los ingresos acumulados por ventas en un determinado periodo).

f) El manejo efectivo de la variabilidad y dependencia dentro de las operaciones y la cadena de suministro permiten un mayor aprovechamiento de los recursos existentes y obtener un mejor retorno de la inversión; las prácticas comunes, como, por ejemplo, la apertura en serie de pequeñas empresas que actúan como intermediarios, no siempre cubren de manera integral las cadenas de suministro y ponen en riesgo la efectividad de la cadena. En la escena de colaboración en línea es necesario contar con un marco conceptual sistémico que permita aprovechar las ventajas tecnológicas.

El objetivo es tratar de difundir los conocimientos, habilidades y actitudes para:

- Analizar su entorno y hacer las modificaciones en las operaciones para protegerlas de la variabilidad y dependencia.

- Desarrollar políticas de ejecución para administrar el nuevo sistema.

- Mantener un proceso de mejora continua.

- Tomar decisiones teniendo en cuenta un enfoque sistémico.

\section{BIBLIOGRAFÍA}

Ballou, Ronald (2004). Logística. Administración de la cadena de sumi-

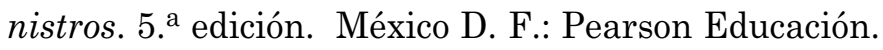

Chase, Richard (2000). Administración de producción y operaciones. Manufactura y servicios. 8. ${ }^{a}$ edición. Bogotá: McGraw-Hill.

Domínguez Machuca, José Antonio et al. (1995). Dirección de operaciones. Aspectos tácticos y operativos en la producción y los servicios. Madrid: McGraw-Hill.

García Cantu, Alfonso (1995). Almacenes, planeación, organización y control. 3. ${ }^{\mathrm{a}}$ edición. México D. F.: Trillas.

Heizer, Jay y Render Barry (2001). Dirección de la producción. 6. ${ }^{a}$ edición. Dos tomos. Madrid: Prentice Hall.

Laseter, Timothy (2000). Alianzas estratégicas con proveedores. Un modelo de abastecimiento equilibrado. Bogotá: Norma. 
Meredith, Jack R. (1999). Administración de las operaciones. Un énfasis conceptual. 2. ${ }^{\mathrm{a}}$ edición. México D. F.: Limusa.

Noori, Hamid y Radford Russell (1997). Administración de operaciones y producción. Calidad total y respuesta sensible rápida. Bogotá: McGraw-Hill.

Schroeder, Róger (1992). Administración de operaciones. Toma de decisiones en la función de operaciones. $3 .^{\mathrm{a}}$ edición. México D. F.: McGraw-Hill. 